\title{
MPIGate: A Solution to use Heterogeneous Networks for Assisted Living Applications
}

\author{
Hugo Cruz-Sánchez, Lionel Havet, Moutie Chehaider \\ INRIA \\ 615, rue du Jardin botanique, \\ 54600 Villers-lès-Nancy, FRANCE \\ Email: Hugo.CruzSanchez,Lionel.Havet,Moutie.Chehaider@inria.fr
}

\author{
Ye-Qiong Song \\ Université de Lorraine - LORIA \\ Campus Scientifique, B.P. 239 \\ 54500 Vandouvre-lès-Nancy, FRANCE \\ Email: song@loria.fr
}

\begin{abstract}
Existing sensors and actuators, that can be used in an AAL (ambient assisting living) environment, work on heterogeneous network protocols, e.g., WiFi, Bluetooth, EIB/KNX and Zigbee. For a given sensor/actuator, the choice of its underlying communication protocol is optimized according to its bandwidth and/or energy needs. However, integrating sensors/actuators of heterogeneous network protocols into an AAL system arises interoperability problems. In this paper, we present MPIGate, a multi-protocol gateway and interface for assisted living applications. Besides its multiple communication drivers for supporting the different protocols, MPIGate also proposes a database for storing the last updated sensor data as well as an user interface for both transparent data access and easy application development. MPIGate has been deployed in a smart home technical test bed at our LORIA labs. We presents some first results and discuss the lessons learnt.
\end{abstract}

Keywords-Assisted living, Health monitoring, Heterogeneous network, Multi-protocol gateway, Middleware

\section{INTRODUCTION}

With the ageing of population, diseases associated to age are growing: living longer increases the probability of suffering from chronic diseases and/or dependency [1]. In order to cope with this situation, health professionals and governments are moving from a hospital-centric care systems to a social care assisted living. Many projects arise to provide new services to patients suffering from chronic diseases, physical or cognitive dependency in order to assist them while staying at home. These projects rely on the emergence of Ambient Intelligence (AI) over this last decade through the growth of Information and Communication Technologies (ICT) in our daily life. Two surveys provides an overview of AI technologies [2] and an outline of smart home projects for health monitoring [3].

Early AAL projects focused on a single infrastructure being either home automation, robotics, sensor networks, actimetry (i.e. monitoring of the daily activities of an elderly for early abnormaly detection) systems or humancomputer interfaces. For instance, home automation system was the first introduced for AI. Technologies supporting home automation have been developed specifically for this market and use either proprietary communications protocols or domain specific standard, e.g. EIB/KNX. As a result, such systems were not deployed at a larger scale due to their cost, the lack of the interoperability with other network standards and the lack of flexibility [4]. However, the sensors and actuators, although initially deployed for home automation, can also be used for AAL. For example, the electricity, water and gas counters may be used as the indicators of the daily activity of the inhabitant. Another example is that the currently available in-home wireless bio-medical sensors (e.g., smart body scale, Blood pressure meter, Oximeter, Glucose monitor, ...) [5] that run on Bluetooth are interesting to be integrated into a global AAL system for monitoring the health evolution of the inhabitant. However its integration still needs extra-engineering efforts. More recently there are also many tentative to use IEEE802.15.4-based wireless sensor networks for AAL [6], [7]. Their effective use is still limited by immature networking technology and lack of the available sensors running on it.

In our opinion, today's assisted living environment must integrate several of these heterogenous infrastructures in a home living environment. The reasons are multiple. The first is to benefit from the wide range of the currently available sensors but not limited to the only sensors supported by a single protocol The second is that the nature of the data and their volume are so different one from another that a single protocol cannot well support them (e.g. data throughput vs. energy consumption). The third is that sensors deployed for other purposes can be useful for AAL applications and their use may reduce drastically the total cost (e.g. home automation sensors for AAL). Using heterogeneous sensors allows to better benefit from their complementarity, achieving thus a more efficient AAL system.

In recent years in France some experiences encouraged by the public administration are trying to combine multiple technologies to offer richer applications for assisted living. Some large-scale demonstrations have been implemented to demonstrate the viability of using combined technologies [8], [9], [10]. The common aspect of all these projects is the use of separate solutions to compose services at application level. None of them integrates implied technolo- 
gies. Indeed, the interconnection with those heterogeneous systems is complex, especially when an AAL application developper should use multi-protocol systems.

In this paper we present MPIGate (Multi Protocol Interface and Gateway for Telecare, Environment Monitoring and Control), a technical solution for system interoperability in an assisted living context by defining a middleware offering modularity and transparent data access. Among other applications, MPIGate can be used in a home assisted living system to integrate multi-platform systems in a unified framework. MPIGate can interconnect multiple networks in one device called MPIGate-box. Information coming from different systems can be processed and used for having a real state of home environment, human activity, etc. The availability of all these variables can be combined to create composite entities to fit the specific application requirements. The gateway uses existing and standard industry infrastructure incorporating wireless sensor networks to complete and propose new sensors and richer applications.

The main contribution of the MPIGate is the definition of an AAL suited gateway architecture allowing to interconnect the heterogeneous networks on the one hand, and to integrate existing middleware solutions on the other hand. In fact, we have successfully implemented three different versions: the first one is the basic one providing data collect and SQL database access, the second one is based on service publisher-subscriber concept by using DDS (Data Distribution Service of OMG, http://portals.omg.org/dds/) for providing quality of service (QoS) support for a subset of AAL applications, and the third one uses ROS (Robot Operating System, http://www.ros.org/wiki/) for easing sensorsrobots interaction in robotic assistance services within an AAL environment. There exist of course also a lot of other approaches such as the web-based ones to realize interoperability like UPnP, or even SIP-based solutions [11]. For further discussions, readers can refer to [12].

The paper is organized as follows. In Section II we describe the requirements of the assisted living application in terms of the system components. The application consists in the daily activity monitoring of an elderly person with chronic diseases and living along at home. In Section III we describe the MPIGate software architecture. Section IV describes the LORIA's Smart Apartment platform ${ }^{1}$ which is used to test MPIGate concept and implementation. In Section $\mathrm{V}$ we present experimental results to demonstrate the feasibility of using the heterogeneous gateway, middleware and interface within the context of AAL application. In Section VI we discuss the implementation process and results to identify important points to be considered in future implementations. Section VII gives concluding remarks.

\footnotetext{
${ }^{1}$ The Smart Appartment at LORIA is part of the CPER MISN Lorraine regional project and INRIA's Personally Assisted Living national project aiming to improve the quality of life for elderly and fragile persons.
}

\section{REQUIREMENTS OF THE ASSISTED LIVING APPLICATION}

The functionalities of an assisted living application can be classified depending on the needs of the user, an elderly/chronic disease person for instance: comfort, security, daily aid/home-support services and/or tele-health services. In all the cases, three key components can be clearly identified:

- environment monitoring and control

- biometric monitoring

- detection of Activities of Daily Life (ADL)

An explanation of these three aspects is given hereafter.

\section{A. Environment monitoring and control}

The need of a permanent knowledge of environment is required to help on inferring the behavior and the activity of the person. Information about the temperature inside and outside of the building, the light on the pieces, etc. can help to explain some behavior or to activate some alarms for example during periods of abnormally hot weather. Environment parameters are available through the home automation network and additional ones can be obtained through IEEE 802.15.4 sensor network when it is required: outside temperature, light on a specific place, etc. As these sensors are independent of the existing infrastructure, the environment can be monitored in a more or less detailed manner to fit the application requirements.

Home automation equipments generally feature dedicated sensors and actuators for home automation and environment control. These sensors include movement detection, temperature, light, electrical and water meters, etc. The actuators are used to regulate current over lights, electrical outlets, doors and windows opening mechanisms, etc.

IEEE 802.15.4 based sensors can be complementary to home automation sensors as they can be installed where the assisted living application needs specific details about the activity of the person or the environmental conditions. Some IEEE 802.15.4 sensors can be used to monitor the state of the doors and windows, to follow the light conditions on a specific room or place, to detect water leak, the $\mathrm{CO}_{2}$ level. A consensus arises in many Ambiant Intelligent platform in favor of EIB/KNX and IEEE802.15.4 [13], [14], [15].

\section{B. Biometric monitoring}

The continuous health monitoring of a person with chronic diseases can help doctors and health professionals to keep this person at home and to avoid long term hospitalizations and/or going to the doctor's clinic for regular consulting. Health monitoring can be implemented with the help of biometric sensors and a system to relay readings to health professionals. In some cases a camera and a system of teleconference may be necessary for remote consulting.

Biometric sensors are based on a variety of communication support. Nowadays the platforms adapted to be used 
are mainly based on WiFi, Bluetooth, IEEE802.15.4/Zigbee and GSM/GPRS standards to forward biometric data to central servers. We pay special attention to the devices using the Bluetooth standard as the Bluetooth Special Interest Group has released in 2008 the Health Device Profile (HDP) [16]. Recently some initiatives converge to the use of IEEE802.15.4/Zigbee-based devices over biometric sensors [17]. These solutions are easily integrated to the Electronic Health Records (EHR) and Personal Health Records (PHR) software. Many devices are available including scale, blood pressure/pulse, temperature, ECG, pulse oximeter, glucometer, among others. These sensors are required in the case of patients receiving treatment for chronic diseases.

\section{Monitoring Activities of Daily Life (ADL)}

Sensors used for ADL monitoring may be connected to different networks and in general they have been designed to fulfill the well defined tasks. For example a movement sensor used in a home automation network is generally to control lights. The data transmitted on the network can be used to locate the activity zone, to infer the activity duration or the trajectory of the person. The combination of data of distributed sensors may be used to infer activity states, intensities, durations and even to predict some states. The ADL monitoring part of the assisted living application can benefit from data coming from all sensors installed on the environment and data circulating in the networks: presence, movement, electric/water consumption, door/window state, shutter window level, etc.

An accurate ADL monitoring requires a maximum data gathering. As already shown, data comes through heterogeneous networks. The interest of MPIGate is that it facilitates the access to the data independently of the communication protocol at lower layers. MPIGate implements a context aware middleware for data abstraction and provides interfaces to facilitate the development of specific applications.

\section{MPIGATE ARChitecture}

In this section we describe the MPIGate software architecture from the functional point of view.

Figure 1 highlights the software components which are describted here:

- Configuration Interface: This is an interface to configure different parameters of the system. By using this interface we can provide and modify necessary information to process the information in the Data Processing Layer.

- User Interface: This provides a graphical user interface for sensor/actuator's state visualization. The interface can be configured by the Configuration Interface to access to any sensor/information or reports generated by the system or to any specific application profile.
- Abstraction: This layer hides the heterogeneity of the system for the users, by providing communication facilities between the Configuration and User Interfaces. The provided API interacts directly with this module.

- Data Processing: This layer is the key part of the system. It process the information collected from the network, saves the results on the database in a local or remote manner. The process to have an homogeneous syntax over all segments of the network is included here.

- Query Manager: This component receives queries from user interface, creates the queries and sends them over the network. A query might be a command to control an actuator or a sensor data request.

- Protocol Abstraction: This provides necessary information about each communication protocol used in the network. Its modularity allows to aggregate necessary protocols for a specific scenario.

- Data Collection: This module collects the data from the network and sends them to the data processing layer. In the scenario described in Section IV it includes necessary background processes to interact with the EIB/KNX, WiFi, Bluetooth and IEEE 802.15.4 over the peripherals of MPIGate.

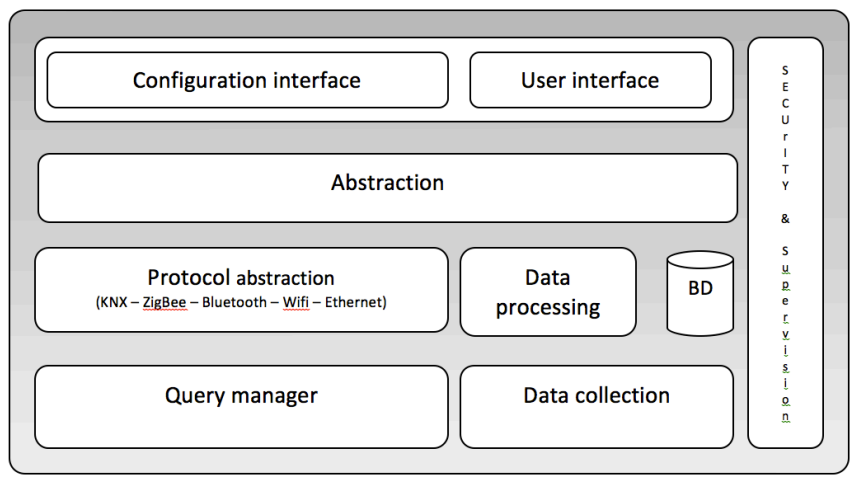

Figure 1. Software architecture of MPIGate

As already mentioned, the abstraction layer can also use any existing middleware. For instance, we have successfully implemented both DDS and ROS. The former for providing real-time QoS management and the latter for easing sensorrobots interaction. So the defined architecture is flexible enough for allowing the plugin of other middleware.

\section{IMPLEMENTATION}

Herein we describe the test used for experimentation and the equipment used which is installed at the LORIA's smart apartment.

\section{A. Experimentation}

This experimentation aims to prove the ability of MPIGate to react with different and heterogeneous technologies and 
to provide a continuous and reliable monitoring of daily activity of persons living at the smart home. We consider three test cases:

- Daily activity monitoring: Analyzing collected data on the gateway allows to follow the movement of a person, his activity, his health and the state of the environment. On mouvement or action detection some ZigBee sensors transmit related data to MPIGate (e.g. movement on the living room, presence of a person on the bed...). By querying the home automation server, MPIGate can monitor the environment and control it through the actuators. The readings generated by the biometric sensors allow to monitor the evolution of the person's health (e.g measuring the weight, blood pressure, pulse and oxygen saturation). Combining all these data provides in real-time a global picture of the smart home's state and its resident activity.

- Detection of an unusual activity: The LORIA's Smart Apartment is equipped with a companion robot that can help the person in some activities (pick-up objects on the floor) and bring augmented sensing capabilities. For example, if the person has passed his habitual time to wakeup or if the intelligent tiles detects a large mass on the ground, the robot will move to the person to check his situation using its embedded camera. This demonstration illustrates the interaction between ZigBee sensors and the robot (WiFi network).

- Environment regulation: This test case presents the interaction of a smart wireless sensor and an home automation actuator through a light regulation system. Using a mobile ZigBee sensor to measure the ambient luminosity at a specific place, the person is able to obtain a sufficient light level that allows him to practice his activities in a comfortable way. This technique avoids a static configuration of the home automation sensors and prevents unnecessary movements of the person to have a higher luminosity.

\section{B. LORIA's smart apartment}

1) Sensors and actuators: A view of the LORIA's smart appartment is shown in Fig. 3. The smart room is equipped with

- A EIB/KNX [18] home automation network. This wired network monitors light, temperature, movement detection (PIR), electric/water consumption and controls lighting, roller blinds, heating, electric door opening. The MPIGate interacts with the KNX equipments through a EIB/USB gateway and the KNXLive! suite [19].

- An IP camera network monitors each room of the apartment. The cameras are PTZ cameras which can be operated through Internet.

- An IEEE802.15.4 sensor network is deployed in the apartment. This network relies on the WaspMote plat-
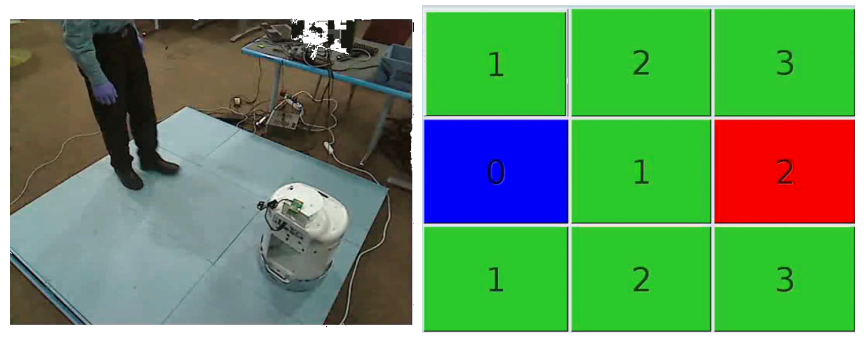

Figure 2. Smart Tiles prototype showing human robot interaction with gradiant propagation.

form [20]. Using ZigBee Pro routing protocol the sensors monitor light, temperature, doors/window opening, presence on chairs/bed/sofas, movement detection (PIR), water consumption. The MPIGate includes a IEEE802.15.4 sink node to interact with this network.

- Biometrics Bluetooth sensors from Boston Life Labs [5]. These sensors features temperature, ECG, weight, pulse and oxygen level monitoring. These sensors are accessed directly through the Bluetooth interface of MPIGate.

- A smart tile floor [21] used for people tracking, walk pattern analysis, fall detection, robot/human interaction, see Fig. 2. This smart floor consists of individual per tile processing units interconnected thought both wired Ethernet and IEEE802.15.4 network.

- Companion robots which communicate through IEEE802.15.4 or WiFi.

A list of components depending on the sensing/acting variable is given in Tab. I.

Table I

SENSORS AND ACTUATORS PRESENT IN THE LORIA'S PLATFORM. THE COMMUNICATION TECHNOLOGIES ARE ON THE LEFT COLUMN.

\begin{tabular}{cl}
\hline & Variable \\
\hline Environment & \\
IEEE 802.15.4 & doors/windows opening, light, temperature, \\
EIB/KNX & lighting control, temperature, blind window control \\
\hline Biometrics & \\
Bluetooth & wrist pulse oximeter, body scale, \\
& wrist blood pressure, ear thermometer \\
\hline Actimetry & \\
IEEE802.15.4 & mouvement detection, Bed/chairs presence \\
EIB/KNX & $\begin{array}{l}\text { lighting control, water meter, electricity meter } \\
\text { blind window control }\end{array}$ \\
\hline
\end{tabular}

The Bluetooth sensors are those provided by Boston Life Labs. MPIGate implements the proprietary protocol using a Bluetooth dongle common to all devices. The original system (HBox hub [5]) uses a dedicated dongle per device and the relay with the EHR/PHR software is performed through the GSM cellular network. MPIGate allows connection to the biometric database using TCP/IP protocol over 
an internet connection. MPIGate can interact with the KNX sensors/actuators over EIB bus through an EIB/USB gateway and using the KNXlive! suite. The system architecture is shown in Fig. 4.

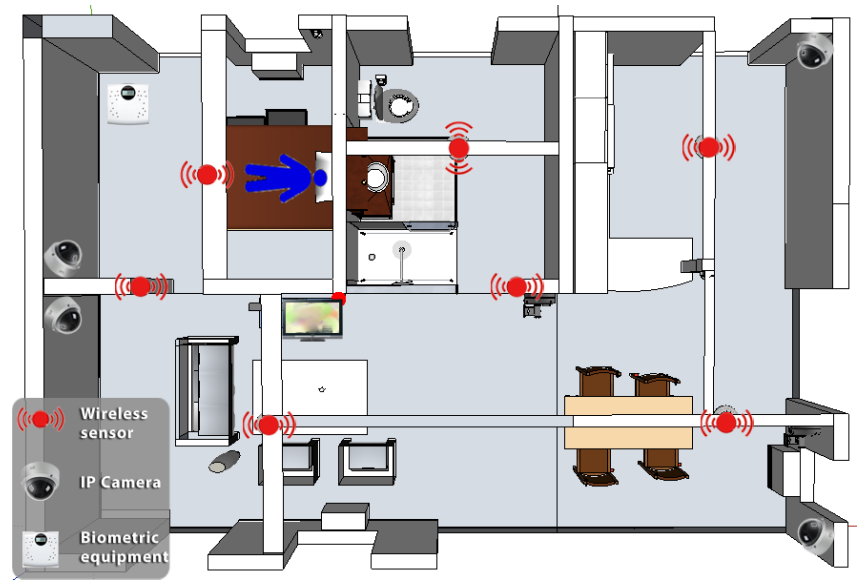

Figure 3. A view of the scenario over the LORIA's smart room.

2) Gateway: The implementation of MPIGate has been realized over $x 86$ architecture using the Linux OS. A prototype has been ported to a Alix card using an embedded Linux OS and running Apache, MySQL and Redis servers. The software interfaces for interaction with user, administrator and developper are:

- User Interface. The user interface framework can be defined by the applications developper depending on the user profile. In general, the user interface allows to access to determined data in a friendly way with different permission levels. The health's professionals may have access to biometric data with some statistic views while relatives may be allowed to follow recent activity data for instance.

- Configuration Interface. It's the interface used to register devices during installation process. The configuration may be performed in parallel with proprietary configuration software, ETS4 [22] for EIB/KNX devices. For other technologies included in MPIGate basic data are needed as location, name, etc. Service discovery is possible if the devices use the standard syntax defined for MPIGate. In IEEE 802.15.4 based devices it is possible as the developper of wireless applications controls the payload of the messages to transmit.

- Application Programming Interface. MPIGate allows to interact with sensors listed through the Configuration Interface by using read () and write () primitives. The applications can be compiled with the MPIGate standard C libraries for local execution. For remote interactions MPIGate allows to use the Application Layer TCP/IP protocol suite to execute local processes, CGI scripts through HTTP protocol for instance.
3) Syntax: An uniformed message syntax has been defined to allow transparent management of messages across the software architecture of MPIGate. The syntax is defined over the intelligent sensor/actuator device when it is possible, on IEEE 802.15.4 devices for instance. For other devices MPIGate implements translation modules depending on the network on which the sensor is located. The physical address of the devices can be used to associate their services. The syntax includes reference marks to separate different readings included in the messages. The defined format is: $\{$ NodeId, PhyAddress, Location, Date, NameOfVariable(1), ValueOfVariable(1),

...

NameOfVariable $(n)$, ValueOfVariable $(n)\}$

where NameOfVariable $(i)$ and ValueOfVariable $(i)$ are a couple of values to identify the kind of variable and its value. The NameOfVariable $(i)$ is defined to $i$ variables used on the system. For instance, the following $i$ variables are used in the LORIA platform:

- 1-19 environmental variables: 1-Temperature, 2-Light, 3-Pressure, 4-Bend, 5,6,7-XYZ acceleration ...

- 20-Battery level of autonomous node

- 30-40 Biometric variables

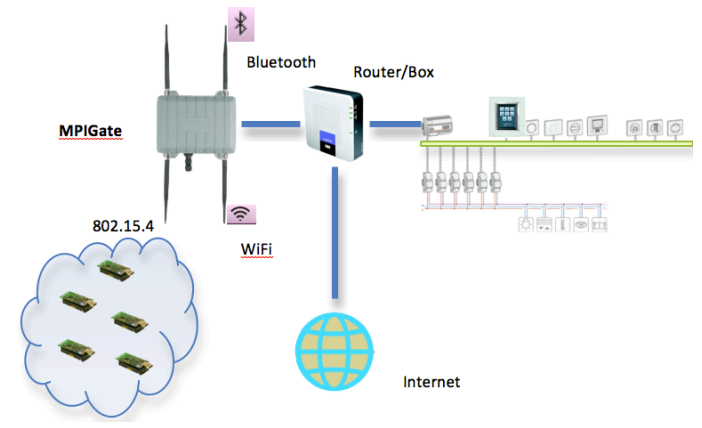

Figure 4. Network architecture of the assisted living environment.

\section{RESULTS}

\section{A. Daily activity monitoring}

For the test we consider a scenario of an elderly woman living at the smart home. She wakes up every day between 8:00 a.m and 8:30 a.m. She suffers from chronic diseases so she needs to daily measure vital signs as blood pressure, pulse and oxygen saturation. These measurements are transmitted on Bluetooth by the biometric sensors to MPIGate database. The data can be consulted by professionals in a remote way using HTTP protocol and a web interface to monitor her health evolution. Examples of some readings are shown in Fig. 5 for blood pressure measurement.

The daily activity of the woman is followed on MPIGate through the heterogeneous sensor's data. A sequence of the data representing the woman's wakeup activity, is illustrated in Fig. 7. 


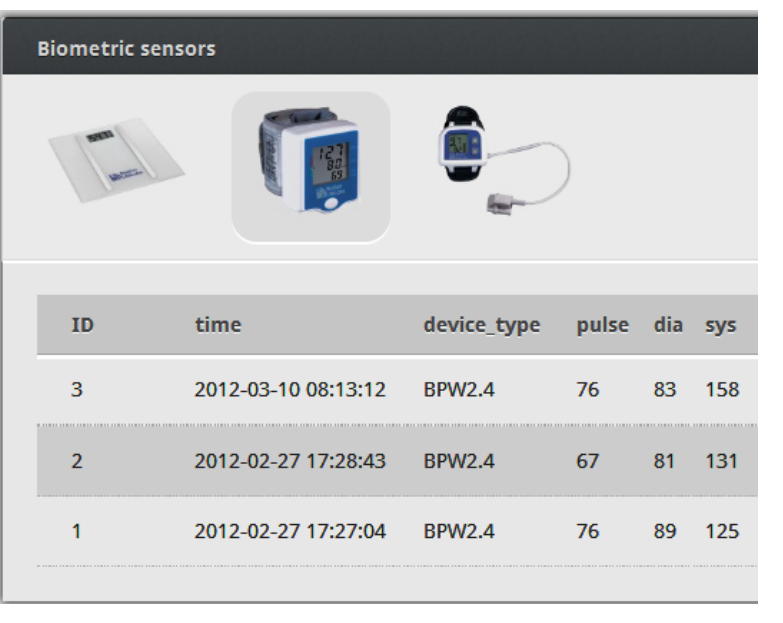

Figure 5. Pressure reading

Finally we check her surrounding environment state. The API of MPIGate allows to read the environment variables by querying the home automation server (e.g shutter state, lamp intensity). The response can be provided in a raw format or using a convenient one, XML for instance, Fig. 6.

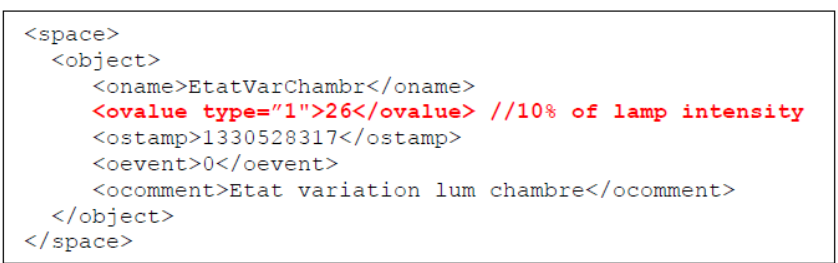

Figure 6. Reading of the lamp intensity

All the captured data of the scenario, are regrouped on the Table II, and analyzed as following. The table is the combination of ZigBee data Fig. 7, home automation data Fig. 6 and Bluetooth data Fig. 5.

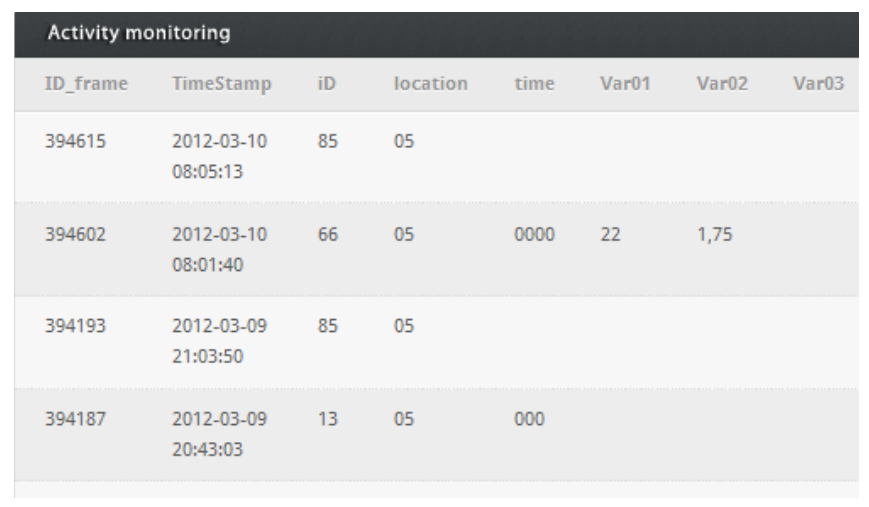

Figure 7. Wake up event

1. The monitored person was present at the bedroom at
Table II

HETEROGENEOUS DATA OF THE OLD WOMAN'S WAKE UP EVENT

\begin{tabular}{|c|c|c|}
\hline Num & Technologies & Captured Data \\
\hline 1 & ZigBee & $\begin{array}{l}\text { Time 20:43:03, Sensor ID 13, Location 5, } \\
\text { Movement detection } 1\end{array}$ \\
\hline 2 & ZigBee & $\begin{array}{l}\text { Time 21:03:50, Sensor ID 85, Location 5, } \\
\text { Bed presence } 1\end{array}$ \\
\hline 3 & ZigBee & $\begin{array}{l}\text { Time 08:01:40, Sensor ID } 66 \text {, Location } 5 \text {, } \\
\text { Temperature } 22 \text {, Luminosity } 1,75\end{array}$ \\
\hline 4 & EIB/KNX & $\begin{array}{l}\text { Lamp intensity } 26 \text {, Shutter state } 1 \text {, Presence } \\
\text { detection } 1\end{array}$ \\
\hline 5 & ZigBee & $\begin{array}{l}\text { Time 08:05:03, Sensor ID 85, Location 5, } \\
\text { Bed presence 0 }\end{array}$ \\
\hline 6 & Bluetooth & $\begin{array}{l}\text { Body scale, Time 08:12:34, Weight } 83 \text {, Type } \\
\text { KG }\end{array}$ \\
\hline 7 & Bluetooth & $\begin{array}{l}\text { Wrist blood pressure, Time } 08: 13: 12 \text {, Pulse } \\
76 \text {, Dia } 83 \text {, Sys } 158\end{array}$ \\
\hline
\end{tabular}

8:40 p.m. last night.

2. She goes to the bed at 9:03 p.m.

3. At 8:00 a.m. the environmental conditions of the room are: Temperature is $22{ }^{\circ} \mathrm{C}$ and Light level is 1.75 $(58 \%)$.

4. Query of the domestic server to check the environment state: Lamp is turned on nightlight, the shutter is open, and a body is present on the room.

5. She wakes up at 8:05 a.m.

6 and 7. Reading of weight and wrist blood pressure from the biometric sensors

\section{B. Detection of an unusual activity}

Following the previous scenario, a robot follows the person's wake up event, by studying his presence on the bed starting from 8:30 a.m and analyzing the transmitted data from the ZigBee sensor installed on the bed to detect his presence (Fig. 7, frame 394615). Then when it reaches an alarm threshold, the companion robot computes a path to the bedroom, see Fig. 8. The robot will provide visual inspection to confirm the current situation or to detect an anomaly on the sensors. The ZigBee sensor is the destination of the robot. Every sensor has its coordinates on the house referenced by his ID and room location. A real demonstration of the test is available on this link http://mpigate.loria.fr/demonstration/interactionRobot.avi.

\section{Environment regulation}

A sample scenario allows to illustrate the interaction of the Zigbee network with the home automation environment, through a light regulation system implemented on MPIGate. The user is able to regulate dynamically his ambient luminosity, using the Zigbee mobile wireless sensor that provides input to act on the lamp regulator for obtaining the required brightness. For example, for a low level of light detected in the living room $(40 \%)$, the person moves the light sensor 


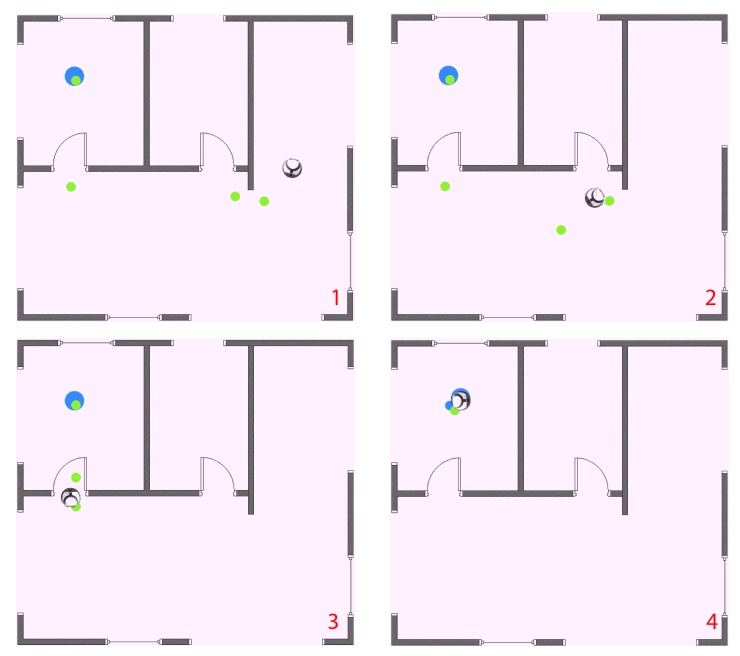

Figure 8. Sequence of the robot's movement showing the trajectory from its initial position to the position of the ZigBee sensor (blue target). At each position reached (green target), the robot discovers its environment and recalculates the path to its destination to avoid obstacles.

at his position and automatically a CGI request will be sent to the domestic server, (see Tab. III), to augment the lamp's intensity and to reach the user desired brightness (e.g. 80\%) in the room.

Table III

MPIGATE SENDDATA API

\footnotetext{
SendData (operation= "1", Login = "user", password= "mpigate", Target= "Variation-salon", value= "191" )

//Operation 1: Write, Login, Password, Target: Actuator name, Value 191: Lamp variation value.
}

In MPIGate, several decisional applications based on SCC (Sense/Compute/Control) pattern, was implemented on the application layer that control and react on the external environment. To develop those applications we are using DiaSuite [23], a Software tools for building pervasive computing applications. On this layer, we defined with the DiaSpec language, a taxonomy that describes the environmental entities as a set of data sources and actuating capabilities, abstracting over devices, whether hardware or software. DiaSuite applications interact with the actual environment through our own APIs, like CGI Request to act on the domestic equipment (see Tab. III). Our current work consists to link the SCC applications to communicate directly with the environment through the middleware layer based on the real-time data distribution service of DDS.

\section{DISCUSSION}

Measurements on our LORIA smart apartment test bed have been carried out showing several interests of the MPIGate design. However, they also allowed us to identify some critical points that must be addressed in our future work.
Firstly, all sensors, with their heterogeneous underlying communication protocols, are successfully accessed, proving thus the interoperability can be effectively achieved using a multi-protocol gateway. For most of the application cases, users can be satisfied by reading periodically or eventtriggered updated and time stamped data stored in the database, which is located inside MPIGate. However, those tests also revealed that for some sensing-control loop applications requiring real-time actuation (e.g. lighting control), the fact that the Zigbee luminosity sensor data should be memorized to the database, and then used by the controller to send actuation command through the home automation network (here EIB/KNX) may introduce excessive delay. This calls for developing primitives at API for also allowing direct data access to some specific sensors and actuators. DDS is an interesting solution. From the gateway middleware design point of view, this requires to define and develop QoS (quality of service) specification in distributed health information systems [24]. An important point considered in future developing work around MPIGate is QoS requirements, like availability and response time. For these raisons and for providing real-time data collecting, we envisaged to use a context-aware middleware [25] to give a dynamic and intelligent QoS to the system. It provides context data and takes into account QoS requirements of the applications.

Secondly, service discovery may be realized for only part of sensors (e.g. Zigbee sensors using their ID or physical address such as MAC address. An unknown ID received by MPIGate may be used to trigger the discovering of a new sensor). How to make it possible for all types of sensors is still an open issue. Our future work will focus on adding service discovering feature to MPIGate, in order to achieve the plug and play until certain degree.

Finally, concerning the IEEE802.15.4-based wireless sensor network part, two problems still remain to solve. One is the energy consumption of the sensor nodes. The current Wasp platform has too high energy consumption. More efficient low-power duty-cycled protocol like X-MAC should be experimented to provide a comparison on the energy efficiency. Another problem is the low throughput that may be insufficient. In fact, as the radio support of Wasp platform is based on the Xbee solution, the sensing is quite limited in throughput. Nevertheless for asynchronous sensing and environment monitoring it's sufficient.

\section{CONCLUSION}

This is an experience to demonstrate the use of distributed sensors over heterogeneous networks for assisted living applications. The use of a multi-protocol gateway with a middleware for abstraction and data processing overcomes many constraints inherent to the use of sensors over varied network technologies so the design of richer applications having information of any sensor is possible. Quality of Service aspects are determinant in application design and 
performance. This aspect must be considered during the Middleware design process.

The architecture of MPIGate allows the applications to interact with any sensor/actuator within the smart environment. The abstraction layer allows to define virtual sensors/actuators by combining available resources. Using this concept MPIGate can be considered as the facade of the smart environment. Applications can be plugged to only by using the API primitives described in this paper. The result is a very simple interface, based on Diasuite, to facilitate applications designed by professionals in the field of assisted living.

Although it seems that all technologies are already available, today it is still difficult to find a comprehensive AAL system allowing its practical use for a large class of elderly. As indicated in[26], one of the reasons is the lack of a common platform. MPIGate has been design to support this point of view. We believe that MPIGate offers a suitable interface to integrate the future UniversAAL specification.

The design and implementation of high level algorithms, such as unusual activity detection by comparing with habitual daily life activities during a longer period, are part of our ongoing work.

\section{REFERENCES}

[1] S. Nourizadeh, C. Deroussent, Y.Q. Song, J.P. Thomesse, "Medical and Home automation Sensor Networks for Senior Citizens Telehomecare", IEEE ICC MAN'2009 Dresden Germany.

[2] D. J. Cook, J. C. Augusto, and V. R. Jakkula. "Ambient intelligence: Technologies, applications, and opportunities". Pervasive and Mobile Computing, 5(4):277- 298, 2009.

[3] D.H. Stefanov, Z. Bien, and W. Bang. "The smart house for older persons and persons with physical disabilities: structure, technology arrangements, and perspectives", IEEE Trans. on Neural Systems and Rehabilitation Engineering, 12(2):228250, june 2004.

[4] Michael C. Mozer. 12 lessons from an adaptive home. In Smart environments: Technologies, protocols, and applications. (eds D. J. Cook and S. K. Das), John Wiley \& Sons, Inc., Hoboken, NJ, USA. 2005.

[5] Boston LifeLabs TeleHealth Solutions. Online http://www.bostonlifelabs.com.

[6] J. Ko, C. Lu, M. Srivastava, J. Stankovic, A. Terzis, and M. Welsh, 'Wireless Sensor Networks for Healthcare", IEEE Proceedings, Vol. 98, No. 11, Nov. 2010, pp.1947-1960.

[7] H. Alemdar and C. Ersoy, "Wireless sensor networks for healthcare: a survey”, Elsevier Computer Networks 54 (2010), pp.2688-2710.

[8] Innovation-Domicile-Autonomie Project. Métropole de Rennes and Rennes University. Online http://www.ida-autonomie.fr/.

[9] The Medical Technology Cluster. "Mobility Project". Online http://en.pole-medical.com/R-D-Project/Maintien-a-Domicile.
[10] Conseil Génégal de la Corrèze. "L’action sociale en faveur du maintien dans le logement". Online http://www.correze.fr/fr/habitat/les-actions-innovantes/.

[11] B. Bertran, C. Consel, W. Jouve, H. Guan and P. Kadionik, "SIP as a Universal Communication Bus: A Methodology and an Experimental Study", in ICC'2010, May 2010, Cape Town, South Africa.

[12] G. S. Blair, M. Paolucci, P. Grace and N. Georgantas "Interoperability in Complex Distributed Systems", in 11th International School on Formal Methods for the Design of Computer, Communication and Software Systems: Connectors for Eternal Networked Software Systems Springer (Ed.), 2011.

[13] F. Cavallo, M. Aquilano, L. Odetti, M. Arvati, and M.C. Carrozza. A first step toward a pervasive and smart zigbee sensor system for assistance and rehabilitation. In Rehabilitation Robotics, 2009. ICORR 2009, pages 632-637, June 2009.

[14] N. Zouba, F. Bremond, M. Thonnat, A. Anfosso, E. Pascual, P. Malléa, V. Mailland and O. Guerin. "A computer system to monitor older adults at home: Preliminary results". Gerontechnology 2009; 8(3):129-139.

[15] C. Reinisch, M. J. Kofler, and W.Kastner. "ThinkHome: A Smart Home as Digital Ecosystem”. In IEEE DEST '10, pages 256-261, April 2010.

[16] Bluetooth Special Interest Group. Bluetooth Specification. Health Device Profile (HDP). June 2008.

[17] ZigBee Alliance. ZigBee Wireless Sensor Applications for Health, Wellness and Fitness. White paper. March 2009.

[18] KNX Association. Online http://www.knx.org.

[19] Automation systems group. Institute of Computer Aided Automation. Vienna University of Technology. KNXLive! suite. Online https://www.auto.tuwien.ac.at/a-lab/knxlive.html

[20] Libelium. http://www.libelium.com/products/waspmote

[21] N. Pépin, O. Simonin, F. Charpillet, "Intelligent Tiles-Putting Situated Multi-Agents Models in Real World", ICAART, 2009.

[22] Engineering Tool Software, ETS4. KNX Association. http://www.knx.org/knx-tools/ets4/description/

[23] Bertran B., Bruneau J., Cassou D., Loriant N., Balland E., Consel C., "DiaSuite: a Tool Suite To Develop Sense/Compute/Control Applications", Elsevier Science of Computer Programming, Fourth special issue on Experimental Software and Toolkits, 2012.

[24] R. Pradeep, G. Weerakkody. "Quality of service management in health care organizations: a case study" 12th IEEE Symposium in Computer-Based Medical Systems, 1999.

[25] S. Nourizadeh, Y.Q. Song, J.P. Thomesse, "CodaQ. A Context-aware and QoS-aware Middleware for Activity Monitoring", ICOST2011, Montreal, Qubec

[26] G. Fagerberg et al., "Platforms for AAL application", in P. Lukowitz, G. Kortuem and K. Kunze (Eds.), EuroSSC 2010, LNCS 6446, pp. 177-201, 2010. 